\title{
Estrategias de interacción en el desarrollo de la destreza productiva del habla del idioma inglés
}

\author{
Interaction strategies in the development of the productive skill of the English \\ language speech
}

Sonia Marcela Suárez Cabrera. ${ }^{1}$, Ruth Patricia Molina Mazón. ${ }^{2}$ \& Sandra Liliana Abarca García. $^{3}$

Recibido: 14-11-2019/Revisado: 04-12-209/Aceptado: 24-12-2019/ Publicado: 04-01-2020

\begin{abstract}
DOI: https://doi.org/10.33262/cienciadigital.v4i1.1077

The objective of the investigation was the application of interaction strategies to improve the development of the productive skill of the speech of the English language in the students of second semester of the Career of Physical Culture of the Faculty of Health Sciences of the National University of Chimborazo. For this, the research was correlational, field research, with methods: inductive, deductive, analytical and synthetic. The sample was represented by 50 students, to whom an intervention plan based on a communicative method was applied, consisting of a set of activities oriented to conversations, dialogues and debates. To obtain the data, an instrument or rubric was applied before and after applying the intervention plan. In the first instance the previous knowledge was evaluated, while in the post test the knowledge was evaluated once the intervention was applied. Three specific and one general hypothesis were formulated based on variables and dimensions in relation to the research context. The data obtained through the intervention were tabulated analyzed, interpreted and subjected to a statistical test, which allowed establishing that the methods and techniques used contributed to the learning; in addition, the productive ability of English language speech was improved and developed by using interaction strategies through active techniques.
\end{abstract}

Keywords: Strategy, Interaction, Development, Productive Skill, English

1 Escuela Superior Politécnica de Chimborazo, Facultad de Salud Pública, Riobamba, Ecuador, sonia.suarez@espoch.edu.ec

2 Escuela Superior Politécnica de Chimborazo, Facultad de Ciencias, Riobamba, Ecuador, ruth.molina@espoch.edu.ec

3 Escuela Superior Politécnica de Chimborazo, Facultad de Salud Pública, Riobamba, Ecuador, sandra.abarca@espoch.edu.ec 


\section{Resumen}

El objetivo de la Investigación fue la aplicación de estrategias de interacción para mejorar el desarrollo de la destreza productiva del habla del idioma inglés en los estudiantes de segundo semestre de la Carrera de Cultura Física de la Facultad de Ciencias de la Salud de la Universidad Nacional de Chimborazo. Para ello, la investigación fue de carácter correlacional, de campo, con métodos: inductivo, deductivo, analítico y sintético. La muestra estuvo representada por 50 estudiantes, a quienes se les aplicó un plan de intervención basado en un método comunicativo, compuesto por un conjunto de actividades orientadas a conversaciones, diálogos y debates. Para la obtención de los datos, se aplicó un instrumento o rúbrica antes y después del aplicar el plan de intervención. En primera instancia se evaluaron los conocimientos previos, mientras que en el post test se evaluaron los conocimientos una vez que se aplicó la intervención. Se formularon tres hipótesis específicas y una general basadas en variables y dimensiones con relación al contexto de la investigación. Los datos obtenidos a través de la intervención, fueron tabulados analizados, interpretados y sometidos a una prueba estadística, que permitió establecer que los métodos y técnicas utilizadas contribuyeron en el aprendizaje; además que se mejoró y se desarrolló la destreza productiva del habla del idioma inglés al usar las estrategias de interacción a través de técnicas activas.

Palabras claves: Estrategia, Interacción, Desarrollo, Destreza productiva, inglés

\section{Introducción}

Maldonado y Romero (2011) en su tesis titulada "Las actividades interactivas que utilizaron los docentes de inglés para el desarrollo de la destreza de hablar en los décimos años de educación básica del Instituto Superior Daniel Reyes, Colegio Nacional San Pablo y Colegio Anexo al ISPED Instituto Alfredo Pérez Guerreo. Provincia de Imbabura durante el año lectivo 2010- 2011 se encontró un enfoque cualitativo y cuantitativo, la metodología fue: descriptiva, de campo, bibliográfica, cualitativa, se obtuvo como conclusiones: Que los docentes no usaron un lenguaje claro al momento de dar una explicación, con lo cual los estudiantes perdieron el interés de aprender el idioma Inglés, no utilizaron actividades interactivas para el desarrollo de la destreza de hablar en sus estudiantes, los docentes no enseñaron a sus estudiantes como establecer y mantener una conversación en Inglés.

Las estrategias fueron definidas como procedimientos (conjunto de pasos, operaciones, habilidades) que un estudiante adquiere y emplea de forma reflexiva, reconocida y premeditada como instrumento flexible para aprender significativamente y tratar de dar solución a un problema que exige el proceso académico. Los objetivos exclusivos de cualquier estrategia de aprendizaje afectaron la forma en que se seleccionó, constituyó o adquirió el nuevo componente e incluso la reforma del cambio expresivo y motivacional (Oxford, 1990).

"La Competencia Estratégica fue una de las competencias que intervino en el uso efectivo de la lengua por parte de una persona. Hizo referencia a la capacidad de servirse de recursos verbales y 
no verbales con el objeto tanto de ayudar la consistencia en la comunicación como de mejorar las falencias que se produjeron en la misma, derivados de algunos vacíos en el conocimiento que se tenía de la lengua o bien de otras condiciones que limitaban la comunicación" (Centro Virtual Cervantes, 2015).

Las competencias se interrelacionaron entre sí; esto hizo referencia a la forma de actuación al momento de hablar, siendo esta un complemento externo donde permitió al estudiante hacer uso de las habilidades de comunicarse, en pocas palabras a la habilidad de hablar en inglés para comunicarse con el mundo exterior.

Se entendió por actividades de interacción aquellas acciones de la lengua principalmente, orales, en las que dos o más estudiantes hicieron el rol de emisor y receptor de mensajes con el propósito de intercambiar información (Centro Virtual Cervantes, 1997).

Según Corrales (2012) las estrategias de interacción fueron formas de enseñanza, donde el estudiante con la intervención de la docente, se constituyó como un sujeto que socializó, discutió, narró, expresó, aprehendió, practicó, explicó, conceptúo, enriqueció, produjo, construyó, generó puntos de vista con respecto a determinados temas, coadyuvando a cumplir con las metas del grupo en el proceso de enseñanza-aprendizaje.

La forma de enseñar a comunicarse en el idioma inglés, fue una concepción coherente del aprendizaje entre el idioma extranjero y la literatura; además, estaba dirigido para que el estudiante desarrolle las destrezas de la lengua en cualquier situación de comunicación formal, informal, académica, científica, cultural (Romero, 2012, p. 12).

La competencia comunicativa, fue uno de los conceptos más importantes en linguística aplicada; tanto en el estudio de la adquisición de segundas lenguas, como a un nivel más práctico en la enseñanza del idioma. En efecto, la competencia comunicativa fue un concepto clave al tratar de responder a las siguientes preguntas: ¿En qué consistió adquirir una lengua?, ¿Qué conocimientos, capacidades o destrezas se necesitaban para hablar una lengua?, ¿Cuál fue el objetivo de la enseñanza de lenguas? La lingüística aplicada muchas veces ha tomado como base teorías de la lingüística teórica o de otras áreas de conocimiento (Cenoz, 2010).

Marín (2013) en las perspectivas propias de las funciones gramaticales, se denominó competencia comunicativa al hecho de expresarse en forma correcta, aplicando el uso de los conectores apropiados para opinar, procesar e interpretar los diferentes tipos de comunicación, teniendo en cuenta no solo lo que se dijo, sino también las implicaciones en el sentido explícito o intencional, lo que el emisor quiso decir o lo que destinatario quiso entender.

La producción verbal fue una de las destrezas más compleja en el proceso comunicativo debido a que el estudiante en un muy poco tiempo tuvo que pensar en lo que va a decir, cómo decirlo de manera clara y sin cometer errores gramaticales o de pronunciación. Adicionalmente, otra dificultad radicó en que se debió no sólo conocer la pronunciación de fonemas sino también el mensaje de la conversación de las personas a quienes el estudiante se dirigió (Gonzáles, 2009).

La comunicación en el habla del idioma inglés es un proceso complejo que se manifiesta en forma espontánea e indispensable, y más complejo es el aprendizaje como lengua extranjera. Entre los problemas más frecuentes que afrontaron los estudiantes es el desarrollo de la destreza productiva 
del habla del idioma inglés, por lo que a través de la presente investigación se induce a comprobar a través de su experiencia personal con los estudiantes de Segundo Semestre de la Carrera de Cultura Física a quienes se les aplicó un plan de intervención para mejorar su habilidad productiva del idioma inglés.

\section{Metodología}

El diseño de la investigación se enmarcó en un contexto cuantitativo, ya que el análisis fue fundamentado a través de datos numéricos obtenidos mediante una encuesta; la misma que fue aplicada a los estudiantes de Segundo Semestre de Cultura Física en el Centro de Idiomas de la Facultad de Ciencias de la Salud (investigación de campo), con la cual se logró valorar la información requerida para la investigación en base a un método estadístico.

La Investigación fue correlacional, ya que se planteó dos variables; por un lado, la variable independiente representada por las estrategias de interacción; mientras que la dependiente son las Destrezas productivas del habla del idioma inglés. Por lo que se planteó una hipótesis a través del efecto que tuvo la variable independiente sobre la variable dependiente, para establecer las respectivas conclusiones.

La investigación fue de carácter educativo, que buscó solucionar el problema a través del diagnóstico aplicado a los estudiantes por medio de preguntas. Para incidir a través de la variable independiente y mejorar el proceso de enseñanza - aprendizaje, se aplicó un plan de intervención a través de un manual denominado Estrategias de Interacción "Aprendo a comunicarme" que permitió el desarrollo de la destreza del habla del Idioma Inglés y que está compuesto por un conjunto de actividades distribuido en lecciones donde se establece la

Se utilizó el método inductivo a través de un análisis ordenado, coherente y lógico del problema de investigación, llegando a establecer leyes generales de comportamiento de los fenómenos a partir del análisis de hechos empíricos. En tanto que el método deductivo permitió que a partir de situaciones generales se logre identificar explicaciones particulares.

Mediante el método analítico - sintético, se determinó la verdad de los hechos reuniendo los elementos que obtuvieron relación lógica entre sí hasta completar y demostrar la verdad del conocimiento.

Se utilizó la técnica de la encuesta y como instrumento el cuestionario, que se aplicó a los estudiantes. La encuesta contenía preguntas fáciles de responder y en especial fueron anónimas para no conservar el anonimato; mientras que el cuestionario permitió obtener información escrita, que evidenciaron las falencias en la aplicación de las estrategias de interacción para desarrollar la destreza productiva del habla del idioma inglés en dos momentos el pre test y post test.

Para comprobar el efecto entre las variables se consideró una población de 50 estudiantes de segundo semestre Carrera de Cultura Física de la Facultad de Ciencias de la Salud UNACH de la ciudad de Riobamba, durante el año 2016. Al ser una población pequeña, se asumió a toda la población como muestra. 


\section{Procedimiento para el análisis e interpretación de resultados}

Los datos obtenidos mediante el instrumento aplicado, fueron tabulados, analizados e interpretados en dos momentos: El pre test y el post test

Para el procesamiento de los datos, se aplicación la prueba estadística Chi cuadrado a cada hipótesis, con el fin de observar la distribución esperada de los datos, que permitió establecer las conclusiones de la incidencia de las dimensiones de la variable independiente con la variable dependiente; establecidas en las siguientes hipótesis

\section{Hipótesis general}

La determinación y aplicación de estrategias de interacción mejoraron la destreza productiva del habla del idioma inglés en los estudiantes de Segundo Semestre de la Carrera de Cultura Física de la Universidad Nacional de Chimborazo en el año 2016.

\section{Hipótesis especificas}

1. El diagnóstico del nivel de la destreza del habla del idioma inglés analizó los métodos y técnicas que se utilizaron en el proceso de aprendizaje del idioma inglés en los estudiantes de Segundo Semestre, Carrera de Cultura Física Facultad de Ciencias de la Salud UNACH, Ciudad Riobamba Año 2016.

2. El desarrollo de estrategias de interacción mejoró la destreza del habla del idioma inglés a través de técnicas activas en los estudiantes de Segundo Semestre, Carrera de Cultura Física Facultad de Ciencias de la Salud UNACH, Ciudad Riobamba Año 2016.

3. La aplicación de las estrategias de interacción desarrolló la destreza productiva del habla del idioma inglés en los estudiantes de Segundo Semestre, Carrera de Cultura Física Facultad de Ciencias de la Salud UNACH, Ciudad Riobamba Año 2016.

4. La evaluación de las estrategias de interacción evidenció el mejoramiento del manejo de la destreza productiva del habla del idioma inglés a través de diálogos, discursos, dramatizaciones creativas en los estudiantes de Segundo Semestre, Carrera de Cultura Física Facultad de Ciencias de la Salud UNACH, Ciudad Riobamba Año 2016.

\section{Resultados}

\section{Aplicación del pre y post test a los estudiantes de Segundo Semestre Carrera de Cultura} Física de la Facultad de Ciencias de la Salud UNACH

\section{Comprobación de la Hipótesis 1}

Hi: El diagnóstico del nivel de la destreza del habla del idioma inglés analizó los métodos y técnicas si se utilizaron en el proceso de aprendizaje del idioma inglés en los estudiantes de Segundo Semestre, Carrera de Cultura Física Facultad de Ciencias de la Salud UNACH, Ciudad Riobamba Año 2016.

Ho: El diagnóstico del nivel de la destreza del habla del idioma inglés analizó los métodos y técnicas no se utilizaron en el proceso de aprendizaje del idioma inglés en los estudiantes de Segundo Semestre, Carrera de Cultura Física Facultad de Ciencias de la Salud UNACH, Ciudad Riobamba Año 2016. 
Modelo matemático

$$
\begin{aligned}
& \mathrm{Hi}=\mathrm{X} 1>\mathrm{X} 2 \\
& \mathrm{Ho}=\mathrm{X} 1<\mathrm{X} 2
\end{aligned}
$$

\section{Modelo estadístico}

$$
\mathrm{X}^{2} \mathrm{C}=\sum \frac{(f 0-f e) x^{2}}{f e}<
$$

\section{Simbología}

$$
\begin{array}{ll}
\chi_{t}^{2}=\text { "Chi" cuadrado calculado } & f_{0}=\text { frecuencia observada } \\
\hline \chi_{t}^{2}=\text { "Chi" cuadrado teórico } & f_{e}=\text { frecuencia esperada } \\
\hline \Sigma=\text { Sumatoria } & \alpha=\text { nivel de significación } \\
\hline \mathrm{IC}=\text { intervalo de confianza } & \mathrm{GL}=\text { grados de libertad }
\end{array}
$$

\section{Nivel de significación}

\section{Regla de decisión}

$$
\begin{array}{ll}
\alpha=0.05 & X^{2} \mathrm{C}>X^{2} t \rightarrow \text { rechaza la hipotesis nula ( Ho) } \\
\text { IC }=95 \% & \mathrm{X}^{2} \mathrm{C}<\mathrm{X}^{2} \mathrm{t} \rightarrow \text { acepta la hipotesis nula ( } \mathrm{Ho} \text { ) }
\end{array}
$$

\section{Zona de rechazo}

Fila de 2 columnas de 3

$$
\mathrm{Gl}=(3-1)(2-1) \quad \mathrm{Gl}=2
$$

$x^{2} \mathrm{t}=3.84$ (valor obtenido en la tabla) Encontramos los valores $x^{2} \mathrm{C}<x^{2} \mathrm{t}$

Tabla 2. Unidad de estudio de la hipótesis 1

\begin{tabular}{|c|l|c|c|c|c|c|c|c|}
\hline \multirow{2}{*}{$\begin{array}{c}\text { UNIDAD DE } \\
\text { ESTUDIO }\end{array}$} & \multicolumn{7}{c|}{$\begin{array}{l}\text { ALTERNATIVAS } \\
\text { o igual a 7 }\end{array}$} & \multicolumn{2}{c|}{ Total } \\
\cline { 3 - 9 } & & Fo & Fe & Fo & Fe & Fo & Fe & \\
\hline 1 & pre test & 2 & 5,5 & 13 & 12,5 & 35 & 32 & 50 \\
\hline 2 & post test & 9 & 5,5 & 12 & 12,5 & 29 & 32 & 50 \\
\hline & TOTAL & $\mathbf{1 1}$ & & $\mathbf{2 5}$ & & $\mathbf{6 4}$ & & $\mathbf{1 0 0}$ \\
\hline
\end{tabular}

Elaborado por: Suárez Sonia, 2016 
Tabla 3. Cálculo de chi cuadrado de la hipótesis 1

\begin{tabular}{|c|c|}
\hline Pre test & Post test \\
\hline$f e_{(11)}=\frac{11 * 50}{100}=\frac{5500}{100}=5.55$ & $f e_{(11)}=\frac{11 * 50}{100}=\frac{5500}{100}=5.55$ \\
$f e_{(25)}=\frac{25 * 50}{100}=\frac{1250}{100}=12.5$ & $f e_{(25)}=\frac{25 * 50}{100}=\frac{1250}{100}=12.5$ \\
$f e_{(64)}=\frac{64 * 50}{100}=\frac{3200}{100}=32$ & $f e_{(64)}=\frac{64 * 50}{100}=\frac{3200}{100}=32$ \\
& \\
\hline
\end{tabular}

Tabla 4. Contingencia de la hipótesis 1

\begin{tabular}{|c|c|c|c|c|c|c|}
\hline $\begin{array}{l}\text { UNIDAD DE } \\
\text { ESTUDIO }\end{array}$ & ALTERNATIVAS & f0 & fe & f0-fe & $(\mathbf{f 0}-\mathbf{f e})^{2}$ & $(\mathbf{f 0 - f e})^{2} / \mathbf{f e}$ \\
\hline \multirow{3}{*}{ Pre Test } & $\begin{array}{l}\text { Promedio mayor o } \\
\text { igual a } 7\end{array}$ & 2 & 5,50 & $-3,50$ & 12,25 & 2,23 \\
\hline & Promedio entre 5 y 6 & 13 & 12,50 & 0,50 & 0,25 & 0,02 \\
\hline & $\begin{array}{l}\text { Promedio menor o } \\
\text { igual a } 4\end{array}$ & 35 & 32 & 3,00 & 9,00 & 0,28 \\
\hline \multirow{3}{*}{ Pros test } & $\begin{array}{l}\text { Promedio mayor o } \\
\text { igual a } 7\end{array}$ & 9 & 5,50 & 3,50 & 12,25 & 2,23 \\
\hline & Promedio entre 5 y 6 & 12 & 12,5 & $-0,50$ & 0,25 & 0,02 \\
\hline & $\begin{array}{l}\text { Promedio menor o } \\
\text { igual a } 4\end{array}$ & 29 & 32 & $-3,00$ & 9,00 & 0,28 \\
\hline \multicolumn{6}{|c|}{ Total $\mathrm{X}^{2} \mathrm{C}$} & 5,06 \\
\hline
\end{tabular}

Elaborado por: Suárez Sonia, 201

\section{Verificación}

Como el $\mathbf{X}^{\mathbf{2}} \mathbf{C}=\mathbf{5 , 0 6}$ fue mayor que el $\mathbf{X}^{\mathbf{2}} \mathbf{t}$ cuyo valor fue 3,84; se encontró en la zona de aceptación, por lo que se rechazó la hipótesis nula de la investigación (Ho), es decir el diagnóstico del nivel de la destreza del habla del idioma inglés analizó los métodos y técnicas si se utilizaban en el proceso de aprendizaje del idioma inglés en los estudiantes de Segundo Semestre, Carrera de Cultura Física Facultad de Ciencias de la Salud UNACH, Ciudad Riobamba Año 2016.

$$
S_{x}=\sqrt{\sum_{i=1}^{n} \frac{\left(x_{i}-\overline{x) 2}\right.}{n-1}}=1.53
$$


Figura1. Campana de Gauss de la desviación típica de la hipótesis 1.

\section{Comprobación de la Hipótesis 2}

Hi: El desarrollo de estrategias de interacción si mejoraron la destreza del habla del idioma inglés a través de técnicas activas en los estudiantes de Segundo Semestre, Carrera de Cultura Física Facultad de Ciencias de la Salud UNACH, Ciudad Riobamba Año 2016

Ho: El desarrollo de estrategias de interacción no mejoraron la destreza del habla del idioma inglés a través de técnicas activas en los estudiantes de Segundo Semestre, Carrera de Cultura Física Facultad de Ciencias de la Salud UNACH, Ciudad Riobamba Año 2016

\section{Zona de rechazo}

Fila de 2 columnas de 5

$$
\mathrm{Gl}=(\mathrm{C}-5)(\mathrm{f}-2) \quad \mathrm{Gl}=4
$$

$x^{2} \mathrm{t}=9.488$ (valor obtenido en la tabla) Encontramos los valores $x^{2} \mathrm{C}<x^{2} \mathrm{t}$

Tabla 5. Cuadro de unidad de estudio de la hipótesis 2

\begin{tabular}{|c|c|c|c|c|c|c|c|c|c|c|c|c|}
\hline \multirow{2}{*}{\multicolumn{2}{|c|}{$\begin{array}{l}\text { UNIDAD } \\
\text { DE ESTUDIO }\end{array}$}} & \multicolumn{10}{|c|}{ ALTERNATIVAS } & \multirow{3}{*}{ Total } \\
\hline & & \multicolumn{2}{|c|}{ Insatisfactorio } & \multicolumn{2}{|c|}{ Regular } & \multicolumn{2}{|c|}{ Bueno } & \multicolumn{2}{|c|}{ Muy bueno } & \multicolumn{2}{|c|}{ Excelente } & \\
\hline & & Fo & $\mathbf{F e}$ & Fo & $\mathbf{F e}$ & Fo & $\mathbf{F e}$ & Fo & $\mathbf{F e}$ & Fo & $\mathbf{F e}$ & \\
\hline 1 & Pre test & 11 & 12 & 20 & 18,5 & 18 & 15 & 1 & 3 & 0 & 1,5 & 50 \\
\hline \multirow[t]{2}{*}{2} & Post test & 13 & 12 & 17 & 18,5 & 12 & 15 & 5 & 3 & 3 & 1,5 & 50 \\
\hline & Total & 24 & & 37 & & 30 & & 6 & & 3 & & 100 \\
\hline
\end{tabular}

Elaborado por: Suárez Sonia, 2017 
Tabla 6. Cálculo de chi cuadrado de la hipótesis 2

\begin{tabular}{|c|c|}
\hline Pre test & Post test \\
\hline$f e_{(24)}=\frac{24 * 50}{100}=\frac{1200}{100}=12$ & $f e_{(24)}=\frac{24 * 50}{100}=\frac{1200}{100}=12$ \\
$f e_{(37)}=\frac{37 * 50}{100}=\frac{1850}{100}=18.5$ & $f e_{(37)}=\frac{37 * 50}{100}=\frac{1850}{100}=18.5$ \\
$f e_{(30)}=\frac{30 * 50}{100}=\frac{1500}{100}=15$ & $f e_{(30)}=\frac{30 * 50}{100}=\frac{1500}{100}=15$ \\
$f e_{(6)}=\frac{6 * 50}{100}=\frac{300}{100}=3$ & $f e_{(6)}=\frac{6 * 50}{100}=\frac{300}{100}=3$ \\
$f e_{(3)}=\frac{3 * 50}{100}=\frac{150}{100}=1.5$ & $f e_{(3)}=\frac{3 * 50}{100}=\frac{150}{100}=1.5$ \\
\end{tabular}

Tabla 7. Contingencia de la hipótesis 2

\begin{tabular}{|c|c|c|c|c|c|c|}
\hline $\begin{array}{c}\text { UNIDAD DE } \\
\text { ESTUDIO }\end{array}$ & ALTERNATIVAS & f0 & fe & f0-fe & $(\mathbf{f 0}-\mathbf{f e})^{2}$ & $(\mathbf{f 0}-\mathbf{f e})^{2} / \mathbf{f e}$ \\
\hline \multirow[t]{5}{*}{ Pre test } & Insatisfactorio & 11 & 12,0 & $-1,00$ & 1,00 & 0,08 \\
\hline & Regular & 20 & 19 & 1,50 & 2,25 & 0,12 \\
\hline & Bueno & 18 & 15 & 3,00 & 9,00 & 0,60 \\
\hline & Muy bueno & 1 & 3 & $-2,00$ & 4,00 & 1,33 \\
\hline & Excelente & 0 & 1,5 & $-1,50$ & 2,25 & 1,50 \\
\hline \multirow[t]{5}{*}{ Post Test } & Insatisfactorio & 1 & 12,0 & $-11,00$ & 121,00 & 10,08 \\
\hline & Regular & 17 & 19 & $-1,50$ & 2,25 & 0,12 \\
\hline & Bueno & 12 & 15 & $-3,00$ & 9,00 & 0,60 \\
\hline & Muy bueno & 5 & 3 & 2,00 & 4,00 & 1,33 \\
\hline & Excelente & 3 & 1,5 & 1,50 & 2,25 & 1,50 \\
\hline & & & & \multicolumn{2}{|c|}{ Total $\mathrm{X}^{2} \mathrm{C}$} & 17,28 \\
\hline
\end{tabular}

Elaborado por: Suárez Sonia, 2017

\section{Verificación}

Como el $\mathbf{X}^{\mathbf{2}} \mathbf{C}=\mathbf{1 7 . 2 8}$ fue mayor que el $\mathbf{X}^{\mathbf{2}} \mathbf{t}$ cuyo valor fue 9.488; se encontró en la zona de aceptación, por lo que se rechazó la hipótesis nula de la investigación (Ho), es decir; el desarrollo de estrategias de interacción si mejoró la destreza del habla del idioma inglés a través de técnicas activas en los estudiantes de Segundo Semestre, Carrera de Cultura Física Facultad de Ciencias de la Salud UNACH, Ciudad Riobamba Año 2016 


$$
S_{x}=\sqrt{\sum_{l=1}^{n} \frac{\left(x_{l}-\bar{x}\right) \overline{2}}{n-1}}=3.39
$$

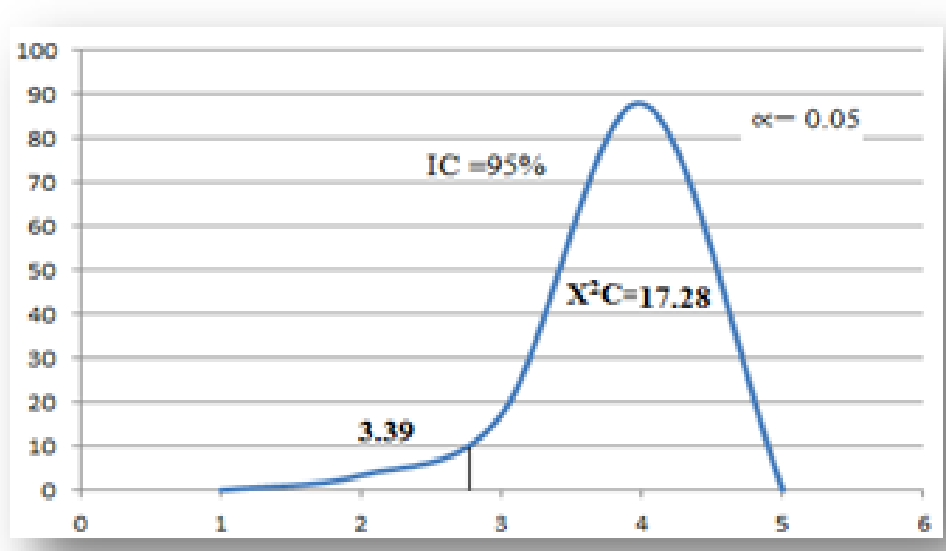

Figura 2. Campana de Gauss de la desviación típica de la hipótesis 2

\section{Comprobación de la Hipótesis 3}

Hi: La aplicación de las estrategias de interacción si desarrolló la destreza productiva del habla del idioma inglés en los estudiantes de Segundo Semestre, Carrera de Cultura Física Facultad de Ciencias de la Salud UNACH, Ciudad Riobamba Año 2016.

Ho: La aplicación de las estrategias de interacción no desarrolló la destreza productiva del habla del idioma inglés en los estudiantes de Segundo Semestre, Carrera de Cultura Física Facultad de Ciencias de la Salud UNACH, Ciudad Riobamba Año 2016.

Tabla 8. Unidad de estudio de la hipótesis 3

\begin{tabular}{|c|c|c|c|c|c|c|c|c|c|c|c|c|}
\hline \multirow{2}{*}{\multicolumn{2}{|c|}{$\begin{array}{l}\text { UNIDAD DE } \\
\text { ESTUDIO }\end{array}$}} & \multicolumn{10}{|c|}{ ALTERNATIVAS } & \multirow[t]{3}{*}{ Total } \\
\hline & & \multicolumn{2}{|c|}{ Insatisfactorio } & \multicolumn{2}{|c|}{ Regular } & \multicolumn{2}{|c|}{ Bueno } & \multicolumn{2}{|c|}{ Muy Bueno } & \multicolumn{2}{|c|}{ Excelente } & \\
\hline & & Fo & $\mathbf{F e}$ & Fo & $\mathbf{F e}$ & Fo & $\mathbf{F e}$ & Fo & $\mathbf{F e}$ & Fo & $\mathbf{F e}$ & \\
\hline 1 & Pre test & 5 & 5,5 & 28 & 25,5 & 16 & 13 & 0 & 4 & 1 & 2 & 50 \\
\hline 2 & Post test & 6 & 5,5 & 23 & 25,5 & 10 & 13 & 8 & 4 & 3 & 2 & 50 \\
\hline & TOTAL & 11 & & 51 & & 26 & & 8 & & 4 & & 100 \\
\hline
\end{tabular}

Elaborado por: Suárez Sonia, 2016 
Tabla 9. Cálculo de chi cuadrado de la hipótesis 3

\begin{tabular}{|c|c|}
\hline Pre test & Post test \\
$f e_{(11)}=\frac{11 * 50}{100}=\frac{5500}{100}=5.55$ & $f e_{(11)}=\frac{11 * 50}{100}=\frac{5500}{100}=5.55$ \\
$f e_{(51)}=\frac{51 * 50}{100}=\frac{2550}{100}=25.5$ & $f e_{(51)}=\frac{51 * 50}{100}=\frac{2550}{100}=25.5$ \\
$f e_{(26)}=\frac{26 * 50}{100}=\frac{1300}{100}=13$ & $f e_{(26)}=\frac{26 * 50}{100}=\frac{1300}{100}=13$ \\
$f e_{(8)}=\frac{8 * 50}{100}=\frac{400}{100}=4$ & $f e_{(8)}=\frac{8 * 50}{100}=\frac{400}{100}=4$ \\
$f e_{(4)}=\frac{4 * 50}{100}=\frac{200}{100}=2$ & $f e_{(4)}=\frac{4 * 50}{100}=\frac{200}{100}=2$ \\
\end{tabular}

Tabla 10. Contingencia de la hipótesis 3

\begin{tabular}{|c|c|c|c|c|c|c|}
\hline $\begin{array}{l}\text { UNIDAD } \\
\text { DE ESTU- } \\
\text { DIO }\end{array}$ & ALTERNATIVAS & f0 & fe & f0-fe & $(\mathbf{f 0}-\mathbf{f e})^{2}$ & $(\mathbf{f 0}-\mathbf{f e})^{2} / \mathbf{f e}$ \\
\hline \multirow{5}{*}{ Pre test } & Insatisfactorio & 5 & 5,5 & $-0,50$ & 0,25 & 0,05 \\
\hline & Regular & 28 & 26 & 2,50 & 6,25 & 0,25 \\
\hline & Bueno & 16 & 13 & 3,00 & 9,00 & 0,69 \\
\hline & Muy Bueno & 0 & 4 & $-4,00$ & 16,00 & 4,00 \\
\hline & Excelente & 1 & 2 & $-1,00$ & 1,00 & 0,50 \\
\hline \multirow{5}{*}{ Post test } & Insatisfactorio & 6 & 5,5 & 0,50 & 0,25 & 0,05 \\
\hline & Regular & 23 & 26 & $-2,50$ & 6,25 & 0,25 \\
\hline & Bueno & 6 & 13 & $-7,00$ & 49,00 & 3,77 \\
\hline & Muy Bueno & 10 & 4 & 6,00 & 36,00 & 9,00 \\
\hline & Excelente & 3 & 2 & 1,00 & 1,00 & 0,50 \\
\hline \multicolumn{6}{|r|}{ Total $\quad \mathrm{X}^{2} \mathrm{C}$} & 19,04 \\
\hline
\end{tabular}

Elaborado por: Suárez Sonia, 2017

\section{Verificación}

Como el $\mathbf{X}^{\mathbf{2}} \mathbf{C}=\mathbf{1 9 , 0 4}$ fue mayor que el $\mathbf{X}^{\mathbf{2}} \mathbf{t}$ cuyo valor fue 9,488; se encontró en la zona de aceptación, por lo que se rechazó la hipótesis nula de la investigación (Ho), es decir; la aplicación de las estrategias de interacción si desarrolló la destreza productiva del habla del idioma inglés en 
los estudiantes de Segundo Semestre, Carrera de Cultura Física Facultad de Ciencias de la Salud UNACH, Ciudad Riobamba Año 2016.

$$
s_{x}=\sqrt{\sum_{i=1}^{n} \frac{\left(x_{i}-\bar{x}\right) 2}{n-1}}=3.70
$$

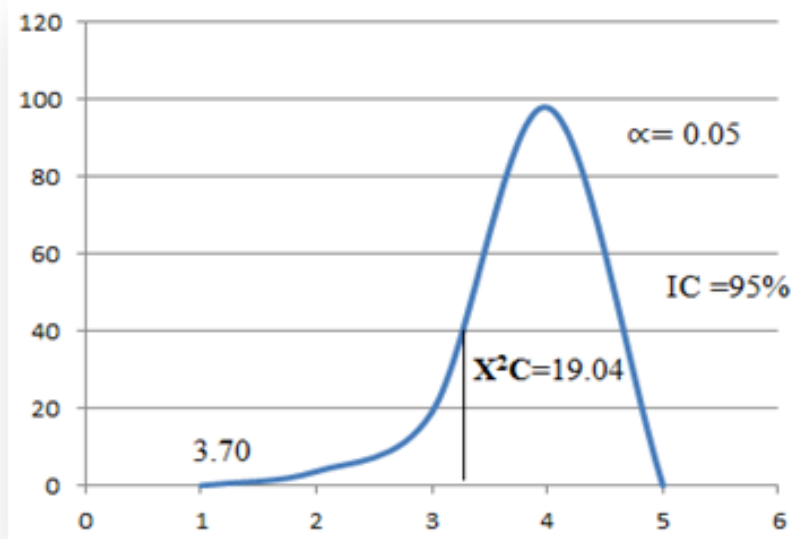

Figura 3. Campana de Gauss de la desviación típica de la hipótesis 3

\section{Comprobación de la Hipótesis 4}

Hi: La evaluación de las estrategias de interacción si evidenció el mejoramiento del manejo de la destreza productiva del habla del idioma inglés a través de diálogos, discursos, dramatizaciones creativas en los estudiantes de Segundo Semestre, Carrera de Cultura Física Facultad de Ciencias de la Salud UNACH, Ciudad Riobamba Año 2016.

Ho: La evaluación de las estrategias de interacción no evidenció el mejoramiento del manejo de la destreza productiva del habla del idioma inglés a través de diálogos, discursos, dramatizaciones creativas en los estudiantes de Segundo Semestre, Carrera de Cultura Física Facultad de Ciencias de la Salud UNACH, Ciudad Riobamba Año 2016.

\section{Zona de rechazo}

Fila de 5 columnas de 2

$$
\mathrm{Gl}=(\mathrm{C}-5)(\mathrm{f}-2) \quad \mathrm{Gl}=4
$$

$x^{2} \mathrm{t}=9.488$ (valor obtenido en la tabla) Encontramos los valores $x^{2} \mathrm{C}<x^{2 \mathrm{t}}$ 
Tabla 11. Unidad de estudio de la hipótesis 4

\begin{tabular}{|c|c|c|c|c|c|c|c|c|c|c|c|c|}
\hline \multirow{2}{*}{\multicolumn{2}{|c|}{$\begin{array}{l}\text { UNIDAD DE } \\
\text { ESTUDIO }\end{array}$}} & \multicolumn{10}{|c|}{ ALTERNATIVAS } & \multirow[t]{2}{*}{ TOTAL } \\
\hline & & \multicolumn{2}{|c|}{ Insatisfactorio } & \multicolumn{2}{|c|}{ Regular } & \multicolumn{2}{|c|}{ Bueno } & \multicolumn{2}{|c|}{ Muy bueno } & \multicolumn{2}{|c|}{ Excelente } & \\
\hline & & Fo & $\mathbf{F e}$ & Fo & $\mathbf{F e}$ & Fo & $\mathbf{F e}$ & Fo & $\mathbf{F e}$ & Fo & $\mathbf{F e}$ & \\
\hline 1 & Pre test & 41 & 28 & 4 & 10 & 2 & 4 & 2 & 6 & 1 & 2 & 50 \\
\hline 2 & Post test & 15 & 28 & 16 & 10 & 6 & 4 & 10 & 6 & 3 & 2 & 50 \\
\hline & Total & 56 & & 20 & & 8 & & 12 & & 4 & & 100 \\
\hline
\end{tabular}

Elaborado por: Suárez Sonia, 2017

Tabla 12. Cálculo de chi cuadrado de la hipótesis 4

\begin{tabular}{|c|c|}
\hline Pre test & Post test \\
\hline$f e_{(56)}=\frac{56 * 50}{100}=\frac{2800}{100}=28$ & $f e_{(56)}=\frac{56 * 50}{100}=\frac{2800}{100}=28$ \\
$f e_{(20)}=\frac{20 * 50}{100}=\frac{1000}{100}=10$ & $f e_{(20)}=\frac{20 * 50}{100}=\frac{1000}{100}=10$ \\
$f e_{(8)}=\frac{8 * 50}{100}=\frac{400}{100}=4$ & $f e_{(8)}=\frac{8 * 50}{100}=\frac{400}{100}=4$ \\
$f e_{(12)}=\frac{12 * 50}{100}=\frac{600}{100}=6$ & $f e_{(12)}=\frac{12 * 50}{100}=\frac{600}{100}=6$ \\
$f e_{(4)}=\frac{4 * 50}{100}=\frac{200}{100}=2$ & $f e_{(4)}=\frac{4 * 50}{100}=\frac{200}{100}=2$ \\
\end{tabular}

Tabla 13. Contingencia de la hipótesis 4

\begin{tabular}{|c|c|c|c|c|c|c|}
\hline $\begin{array}{l}\text { UNIDAD DE } \\
\text { ESTUDIO }\end{array}$ & ALTERNATIVAS & f0 & fe & fo-fe & $(\mathbf{f 0}-\mathbf{f e})^{2}$ & $(\mathbf{f 0}-\mathbf{f e})^{2} / \mathbf{f e}$ \\
\hline \multirow{5}{*}{ Pre Test } & Insatisfactorio & 41 & 28 & 13,00 & 169,00 & 6,04 \\
\hline & Regular & 4 & 10 & $-6,00$ & 36,00 & 3,60 \\
\hline & Bueno & 2 & 4 & $-2,00$ & 4,00 & 1,00 \\
\hline & Muy bueno & 2 & 6 & $-4,00$ & 16,00 & 2,67 \\
\hline & Excelente & 1 & 2 & $-1,00$ & 1,00 & 0,50 \\
\hline \multirow{5}{*}{ Pros Test } & Insatisfactorio & 15 & 28 & $-13,00$ & 169,00 & 6,04 \\
\hline & Regular & 16 & 10 & 6,00 & 36,00 & 3,60 \\
\hline & Bueno & 6 & 4 & 2,00 & 4,00 & 1,00 \\
\hline & Muy bueno & 10 & 6 & 4,00 & 16,00 & 2,67 \\
\hline & Excelente & 3 & 2 & 1,00 & 1,00 & 0,50 \\
\hline \multicolumn{6}{|c|}{ Total $\mathrm{X}^{2} \mathrm{C}$} & 27,60 \\
\hline
\end{tabular}

Elaborado por: Suárez Sonia, 2017 


\section{Verificación}

Como el $\mathbf{X}^{2} \mathbf{C}=\mathbf{2 7 , 6}$ fue mayor que el $\mathbf{X}^{2} \mathbf{t}$ cuyo valor fue 9.488; se encontró en la zona de aceptación, por lo que se rechazó la hipótesis nula de la investigación (Ho), es decir; la evaluación de las estrategias de interacción si evidenció el mejoramiento del manejo de la destreza productiva del habla del idioma Inglés a través de diálogos, discursos, dramatizaciones creativas en los estudiantes de Segundo Semestre, Carrera de Cultura Física Facultad de Ciencias de la Salud UNACH, Ciudad Riobamba Año 2016.

$$
s_{x}=\sqrt{\sum_{i=1}^{n} \frac{\left(x_{i}-\bar{x}\right) 2}{n-1}}=7.62
$$

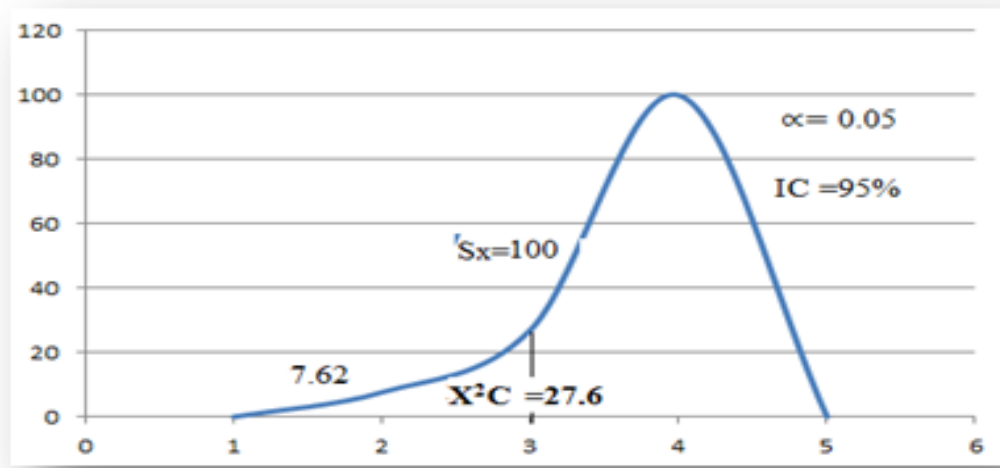

Figura 4. Campana de Gauss de la desviación típica de la hipótesis 4

\section{Conclusiones}

El entendimiento mejoró en cuanto a ideas, nuevo vocabulario, producción de diálogos y conversaciones con menor número de errores gramaticales; mientras que, en la pronunciación, el desenvolvimiento mejoró en el uso correcto de reglas gramaticales, acento, ritmo, coherencia, cohesión, sintaxis y fluidez a través de los conocimientos adquiridos en la aplicación de estrategias de interacción para mejorar la destreza productiva del habla del idioma inglés.

La importancia de la aplicación del método comunicativo, denota que la aplicación de estrategias de interacción, ayuda en la mejorara del desarrollo de la destreza productiva del habla del idioma inglés, ya que resultó eficiente el apoyo al aprendizaje de conocimientos mediante métodos y técnicas activas tales como: pronunciación, gramática, estructura de diálogos, conversaciones, discusiones, dramatizaciones creativas como parte del desarrollo indispensable para su aprendizaje.

El estudio de las estrategias de interacción conjuntamente con su aplicación, permitió que los estudiantes hayan obtenido un cambio significativo en el mejoramiento de la destreza productiva 
del habla del idioma inglés en un 5,06 \%, es decir que con la aplicación del manual mejoró la producción oral.

\section{Referencias bibliográficas}

Centro Virtual Cervantes. (1997). Conceptualización. Obtenido de Actividades y estrategias de interacción:

http://cvc.cervantes.es/ensenanza/biblioteca_ele/diccio_ele/diccionario/activestrategintera ccion.htm

Centro Virtual Cervantes. (2015). Competencias Comunicativas. Recuperado el 6 de enero de 2014, de definiciones:

http://cvc.cervantes.es/ensenanza/biblioteca_ele/diccio_ele/diccionario/enfoquecomunicat ivo.htm

Cenoz, L. (2010). El concepto de competencia comunicativa. Revista de Enfoque comunicativo, $1-10$.

Corrales P, E. (2012). ESTRATEGIAS DE INTERACCION GRUPAL EN EL AULA. Obtenido de concepto: http://mdctb.blogspot.com/2012/07/estrategias- de-interaccion-grupal-enel_17.html

Gonzáles, D. (2009). Estrategias de aprendizaje para el desarrollo de la producción oral en la Licenciatura en Lenguas Modernas de la Pontificia Universidad Javeriana. BogotáColombia: Pontificia Universidad Javeriana facultad de comunicación y lenguaje licenciatura en lenguas modernas.

Maldonado, M. D., \& Romero, M. J. (2011). Las actividades interactivas que utilizan los docentes de inglés para el desarrollo de la destreza de hablar en los décimos años de educación básica del instituto superior Daniel Reyes, Colegio Víctor Mideros, colegio nacional san pablo y colegio anexo al ISPED Instituto Alfredo Pérez Guerrero [Pregrado, UNIVERSIDAD TÉCNICA DEL NORTE]. http://repositorio.utn.edu.ec/bitstream/123456789/2042/1/05\%20FECYT\%201181\%20T ESIS.pdf

Marín, R. (30 de marzo de 20013). Competencias Comunicativas. Obtenido de Respuesta en línea: https://rafaelmarin95.wordpress.com/2013/03/30/competencia-comunicativa/

Oxford, R. (1990). Diagrama de las Estrategias Cognitivas. Boston: Heinle and Publishers.

Romero, D. (2012). El método comunicativo en el aprendizaje del idioma inglés y la propuesta de una guía didáctica para la utilización del método comunicativo para mejorar el aprendizaje del idioma inglés en los/las estudiantes del décimo año del colegio nacional Tarqui. Quito- Ecuador: Universidad Central del Ecuador. 


\section{PARA CITAR EL ARTÍCULO INDEXADO.}

Suárez Cabrera, S. M., Molina Mazón, R. P., \& Abarca García, S. L. (2020). Estrategias de interacción en el desarrollo de la destreza productiva del habla del idioma inglés. Ciencia Digital, 4(1), 126-141. https://doi.org/10.33262/cienciadigital.v4i1.1077

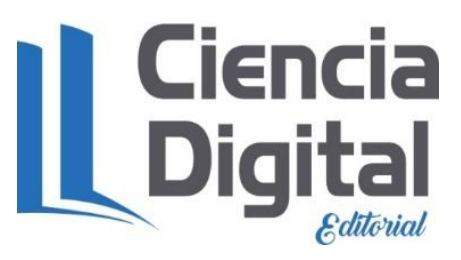

El artículo que se publica es de exclusiva responsabilidad de los autores y no necesariamente reflejan el pensamiento de la Revista Ciencia Digital.

El artículo queda en propiedad de la revista y, por tanto, su publicación parcial y/o total en otro medio tiene que ser autorizado por el director de la Revista Ciencia Digital.
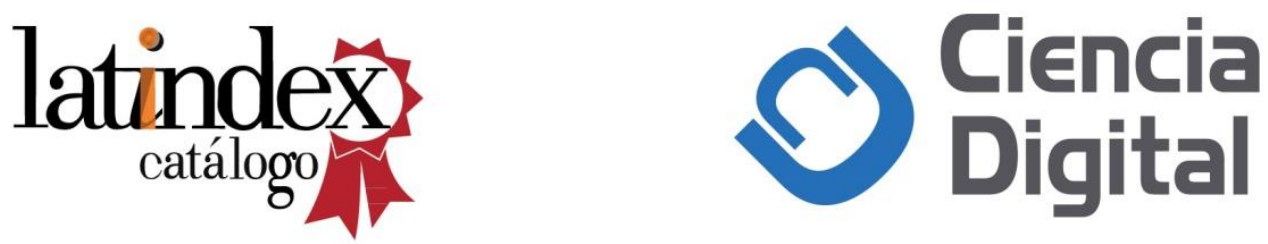